\title{
Design and Realization of Intelligent Warehouse Based on Bluetooth Communication
}

\author{
Jingnan $\mathrm{MA}^{1}$, Chao $\mathrm{SHAO}^{2}$, Jia LI' ${ }^{1}$, Shaohong $\mathrm{LAN}^{2}$, Tao YANG ${ }^{2}$ \\ ${ }^{1}$ Technical Institute of Air Force Engineering University, Xi'an China \\ ${ }^{2}$ Aerospace Institute of Air Force Engineering University, Xi'an China \\ 52389425@qq.com
}

\begin{abstract}
Keywords: MCU, Bluetooth Communication, Master Controller.
Abstract. With the development of computer technology, communication technology, automatic control technology, life and production management more intelligence and information. The design of intelligent warehouse consists of three parts, the entrance guard control, the temperature and humidity control, the fire monitoring and the fire warning system, the data is transmitted via Bluetooth to master controller, enabling intelligent warehouse management.
\end{abstract}

\section{Introduction}

Rapid development of science and technology, lead the digitization, intelligentialize and networking has become the inevitable trend of human society progress. Continuous development of computer science, communications technology and automatic control technology on amount of the fast development of information technology, such technology and skills had been integrated into people's daily life. Therefore, it catalyze the industrial boom of intelligent housing system 【IHS】 revolution during last several years, as an benefit, application of intellectualization and networking has been a trend in warehouse management field.

Some of the problems frequently made in application of wired communications, such as wire range of complex lines and other electrical hazards, wireless communications transmission as an efficient data transmission, which has been established as an irreplaceable role of 【IHS 】, industrial control and other aspects. The advantages of low cost, less power consumption and succinct configuration, which enable wireless data transmission plays an important role in many industry fields. Bluetooth communication in wireless communication system is an important technology, but also the researchers of all sectors are focus on it in recent years.

\section{The Structure and Principle of the System}

With the development of the Internet of Things, the warehouse management is being intelligent and networking. The design of intelligent warehouse's environmental parameters is through the Bluetooth wireless communications transferring to the users, and the users can manage the temperature, humidity, entrance guard and other aspects of the warehouse through the Bluetooth wireless networking.

The block diagram of the intelligent warehouse is shown in Fig. 1.

Users can take advantage of PC and CPU master controller control three functions of intelligent warehouse via Bluetooth networks, including the entrance guard systems, temperature and humidity monitor systems as well as the emergency systems. The data of these three parts can also be passed back to PC and CPU by Bluetooth network.

\section{System Design Plan}

The Design of Master Controller. The master controller of this design uses the device for IAP15F2K61S2, which the STC company launched 8051 MCU in 2011. The MCU has a single clock/machine cycle (1T), which is characterized by high speed, high reliability, low power consumption and strong anti-jamming. Its greatest feature is that can work without external crystal and 
external reset circuit, because it integrated precision $\mathrm{R} / \mathrm{C}$ clock circuit, and its internal clock can be selected from $5 \mathrm{MHz}$ to $35 \mathrm{MHz}$. In addition, this device has 8-channel 10-bit high-speed ADC, and its speed is 300ksps. The device has six timers, which has two 16-bit reload timers T0 and T1. Its clock can be programmable output. This device's programmable mode is ISP or IAP, without programming device or emulator. The design's program flow chat is shown in Fig. 2.

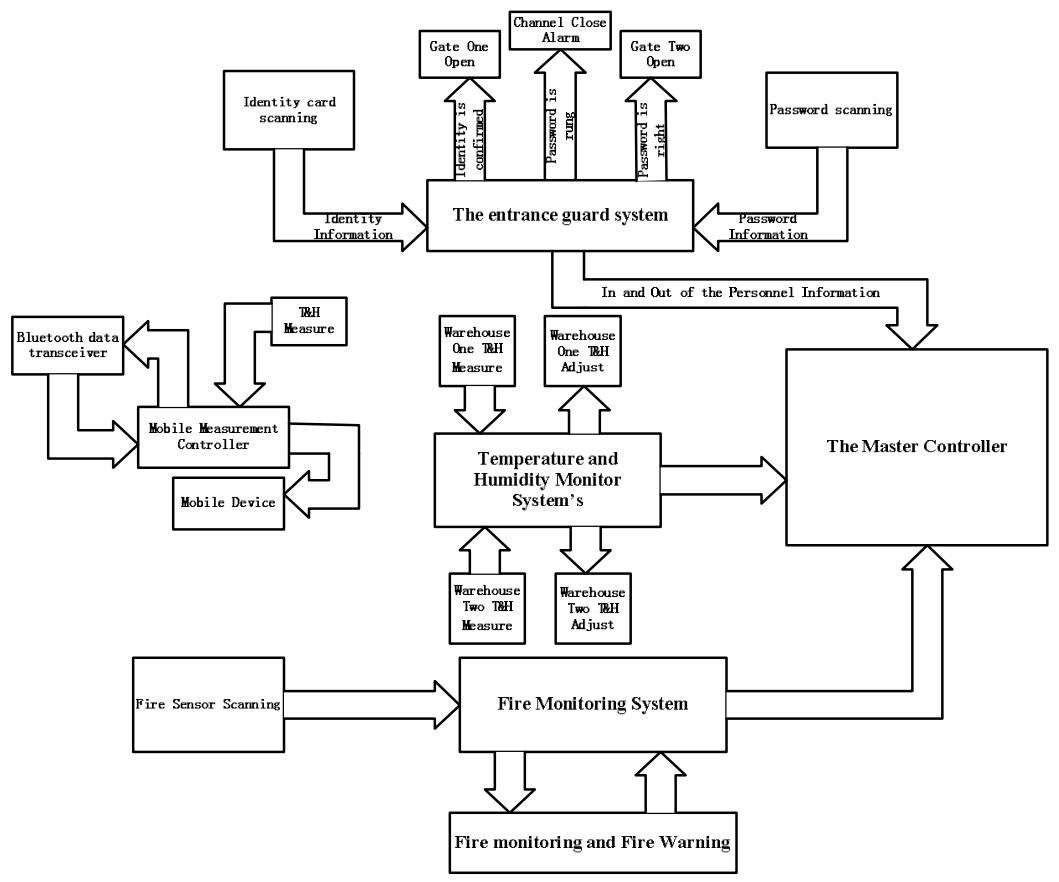

Fig. 1 The block diagram of the intelligent warehouse

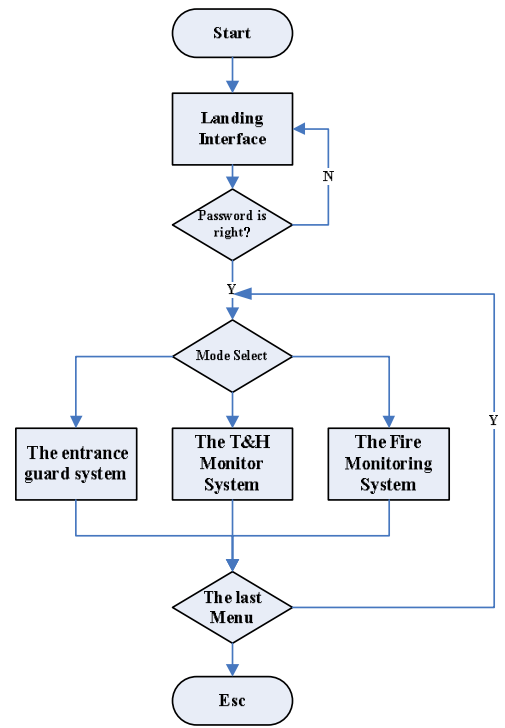

Fig. 2 Program Flow Chat Entrance guard system's Design. The entrance guard systems including identity and password door, which can manage and register the staff accessing. If the card closes match, the security person can open the door, otherwise the unfamiliar person will be isolated, and the dangerous person will be imprisoned to police.

The identity card work by RFID. RFID (Radio Frequency Identification) technology, also known as radio frequency identification, is a communication technique, by electrical signals identifies specific targets and associated write data without recognition system between the target and the establishment of a specific mechanical or optical contact. RF is typically microwave, $1 \sim 100 \mathrm{GHz}$, suitable for short-distance communication identification. The radio frequency identification module in this design can only be read EM4100 read-only series tag cards for $125 \mathrm{KHz}$. Each tag card contains a unique identifier, which is the one of $2^{40}$, or has $1,099,511,627,776$ possible combinations. After reading the tag card's data from the RFID radio frequency identification card (RFID reader), transmitted via serial interface, its serial port baud rate is $2400 \mathrm{bps}$.

When the RFID radio frequency identification card and label card placed to work within the effective reading range, a unique tag number in the form of 12-bit ASIC II string will sent to the controller via serial communication. Its data in the form is shown in Table 1.

Table 1 Label Card Data Form

\begin{tabular}{|l|l|l|l|l|l|l|l|l|l|l|l|}
\hline Start Bit & \multicolumn{9}{|c|}{ The Only Number } & Stop Bit \\
\hline 0x0A & Bit 1 & Bit 2 & Bit 3 & Bit 4 & Bit 5 & Bit 6 & Bit 7 & Bit 8 & Bit 9 & Bit 10 & 0x0D \\
\hline
\end{tabular}

The Password Door is to determine the identity of the three types person's password authorized by the controller, it is mainly determined by the software.

Temperature and Humidity Monitor System's Design. The temperature and humidity sensor of this design is used DHT11 digital temperature and humidity sensor which made in China. This device has been calibrated digital signal output of the temperature and humidity complex sensor, using a dedicated digital modules collection technology and temperature and humidity sensing technology. The sensor contains a resistive humidity element and a NTC temperature measurement element, connecting with a high-performance 8-bit MCU. The sensor is calibrated in the extremely precisely humidity calibration 
lab, and the calibration factor in the form of program stored in the OTP memory, which the sensor called in the process of detecting signals. The sensor uses single-wire serial interface, and the signal transmission distance is up to 20 meters. When the sensor connected to the circuit, you can use a suitable pull-up resistor according to the actual situation when the transmission distance is longer than 20 meters; otherwise you can use a $5 \mathrm{~K}$ pull-up resistor.

DHT11 has a single-wire bidirectional serial interface, which using a single data bus format. The communication speed is about $4 \mathrm{~ms}$ per time, and the data contains decimal part and integer part. A complete data transmission is 40 bit, high-first-out, and the data format is: 8 bit humidity integer data + 8 bit humidity decimal data +8 bit temperature integer data +8 bit temperature decimal data +8 bit checksum. If the transmission data is right, the checksum is the sum of the last 8 bit for " 8 bit humidity integer data +8 bit humidity decimal data +8 bit temperature integer data +8 bit temperature decimal data ". DHT11 Sequence diagram of the data transferring process shown in Fig. 3.

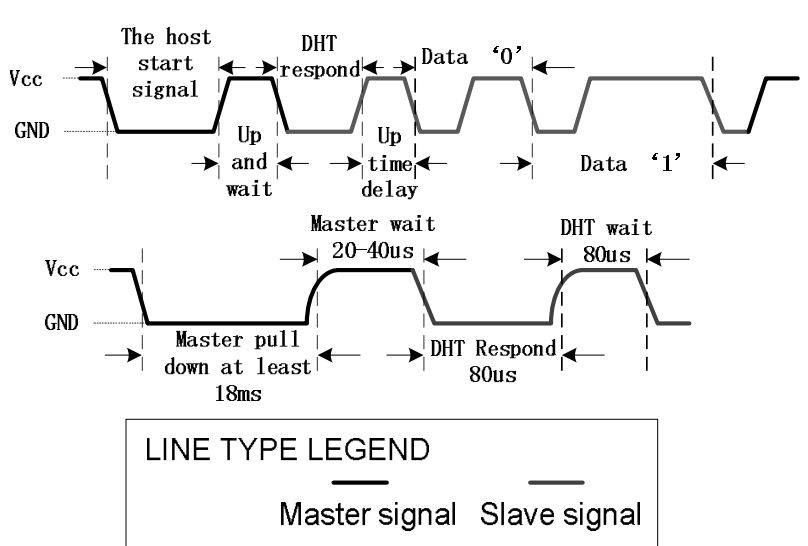

Fig. 3 DHT11 Sequence diagram
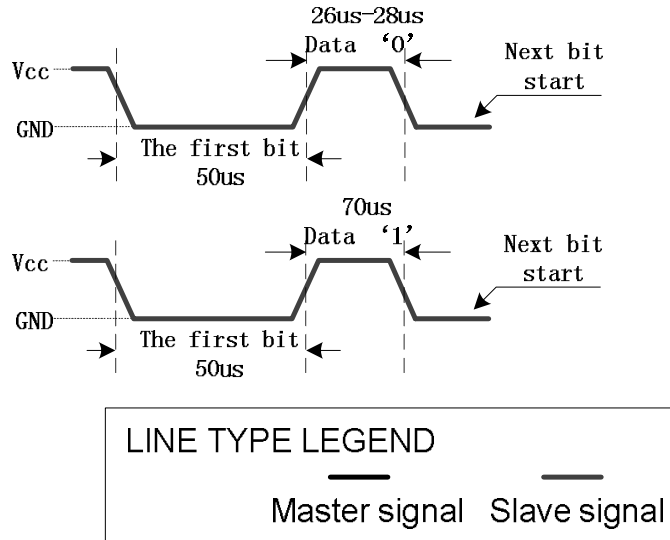

Fig. 4 DHT11 data format

After the controller sends a start signal, DHT11 transforms from a low-power mode to the high speed mode, the bus will pull down waiting for DHT11 response. At this time, the low level time of the bus must be longer than $18 \mathrm{~ms}$, in order to ensure DHT11 detected the start signal. Than the high level time of the bus waiting for 20 40 us, DHT11 will be ready to get the response signal. When DHT11 receive the start signal, it will send low level signal for 80 us, than hold high level for 80us waiting for data transmission. When sending the 40 bit data and triggering the signal acquisition once, the user can choose reading the part of the data. In slave mod, when DHT11 received the start signal, it will trigger the signal humidity acquisition once. If DHT11 don't receive the start signal, it will not take the initiative to collect the temperature and humidity data, and it will convert to low-speed mode after collecting data.

During the processing of sending data, each bit of data begins with 50us low level. And the time of the high level is determined that the data is 0 or 1. The format shown in Fig.4.

Fire Monitoring System's Design. Fire monitoring system is to detect a fire and timely put out a fire. The system contains two sections. One section is sentinel surveillance, which is a fire warning array of the flame sensor and smoke sensor, it can detect real-time whether there occurred fire in the warehouse.

The other section is the emergency car which can always monitor the fire. This car also equipped DHT11 temperature and humidity sensor, which can measure the gradient change of the temperature and humidity of the warehouse, it also could be emergency standby measuring device.

Bluetooth System's Design. The Bluetooth system of this design is based on the British CSR's BlueCore4-Ext chip, follow V2.1 + EDR Bluetooth specification. CSR's BlueCore4-Ext device implements the Enhanced Data Rate (EDR) Bluetooth specification, allowing it to deliver data rates that are 3-times faster than V1.2 Bluetooth devices. This transfer rate also means that, for a given amount of data, the radio will be active up to 3 times less than a v1.2 radio, reducing power consumption. 
The physical characteristics of the module are shown in Table 2, and the recommended electrical specifications are shown in Table 3.

Table 2 The physical characteristics of Bluetooth module

\begin{tabular}{|c|l|}
\hline Physical Characteristics & Parameters \\
\hline $\begin{array}{c}\text { Operating Frequency } \\
\text { Band }\end{array}$ & $2.4 \mathrm{GHz} 2.48 \mathrm{GHz}$ unlicensed ISM band \\
\hline Bluetooth Specification & V2.1+EDR \\
\hline Output Power Class & Class 2 \\
\hline Operating Voltage & $3.3 \mathrm{~V}$ \\
\hline Host Interface & USB $1.1 / 2.0$ or UART \\
\hline Audio Interface & PCM interface \\
\hline Flash Memory Size & $8 \mathrm{Mbit}$ \\
\hline Dimension & $27 \mathrm{~mm}(\mathrm{~L}) \times 13 \mathrm{~mm}(\mathrm{~W}) \times 2 \mathrm{~mm}(\mathrm{H})$ \\
\hline
\end{tabular}

Table 3 Recommended Operating Conditions

\begin{tabular}{|l|l|l|}
\hline Operating Condition & Min & Max \\
\hline Operating temperature range & $-40^{\circ} \mathrm{C}$ & $+150^{\circ} \mathrm{C}$ \\
\hline Guaranteed RF temperature range & $-40^{\circ} \mathrm{C}$ & $+150^{\circ} \mathrm{C}$ \\
\hline Supply voltage: VBAT & $2.2 \mathrm{~V}$ & $4.2 \mathrm{~V}$ \\
\hline
\end{tabular}

\section{Problems and Solutions}

Problems. When designing and developing wireless transmission and control module of the intelligent warehouse, we found that sometimes data transmission will still be periodic problems of instability, even when the power supply is stable, communication distance is moderate. For example, when the MCU collected the temperature and humidity data, which is sent to the computer through a Bluetooth module, the computer will receive the error data or even lost. But when the computer sends instructions to the remote control car, it will not be any errors. After testing, the circuit components and connections are not unusual, and the program has no obvious errors.

Problems Analysis. When testing the power supply voltage, we found no abnormalities, however when the testing nodes close to the Bluetooth module, circuit parameters appeared a cyclical fluctuations, especially peripheral high-power electric devices working, wireless data transmission appears obvious mistakes. In the system, although there are big power plants which are heating tube and cooling module, but because of the device for the independent power supply, does not affect communication circuit. In contrast, for the motor power consumption devices, although its power rating in the range of power supply, but it has a larger inductance, when the electric motor rotating moment, it can cause circuit parameters have great fluctuation, which affect the stability of Bluetooth data transmission. Test found that data transmission error is related to the motor working state.

When the relay and motor power is on power or no instantly, they all can generate strong magnetic field, which can interfere with the wireless signal transmission. In the hardware system of the intelligent warehouse, there are multiple relays, motor powers and so on, which can generate large magnetic field, such as the complex magnetic field to a certain extent can produce interference to radio communication.

The above two reasons to a certain extent, lead to the Bluetooth communication is not stable, however, it does not explain the computer as a sender. Bluetooth communication itself has a function of error correction that will automatically judge the correctness of the receiving data, and the error data packet loss and resend, so as to ensure the zero error rate of Bluetooth communication. In addition, Bluetooth communication also automatically converts a long data to a shorter data frames to send, in order to reduce the packet loss caused by the problem of low efficient transmission. Just because of this feature, when applying the Bluetooth communication module, if don't accept the appropriate processing, the transmission data will appear errors because the complete data is separated. For example, send password data ABCDEFG1234567, received two ABCDEFG and 1234567 groups of 
data, if identify respectively, the password must be wrong. In the intelligent warehouse system, because the computer data sent to the remote control car in short instructions, so there will be no data errors caused by data separated.

Solution. According to electromagnetic environment interference, we can deal with device which is the relay and motor producing interference for electromagnetic shielding. According to the abnormal voltage circuit, we can connect the parallel capacitor on the both ends of the motor to produce the coil inductive reactance and reduce the fluctuation of circuit parameters. According the separated data, we can recover the received data before processing.

Above three interference factors involved is not independent, also influence each other. Due to the high inductive reactance in the intelligent warehouse, it can produce the strong electromagnetic interference. On the other hand, it can lead to larger circuit parameters fluctuation. Accordingly the Bluetooth module power supply is not stable, eventually resulting in abnormal communication. These problems are relatively hidden, if not into the specific working condition, the error will not appear timely. Mentioned in this paper, the problem is not only appeared in the Bluetooth module communication, also appeared in other wireless communication. Without considering the effects of the problem, take corresponding measures, the system stability will be reduced, affect the system's overall performance. If the problems appeared in the industrial control fields, and even serious hidden trouble will be produced.

\section{Conclusions}

In addition to the design of the intelligent warehouse management system can be used in the equipment library, can also be used for other types of warehouses, farm and so on need to adjust temperature and humidity. When using can reduce the workload of management, and to make weapons and equipments management and maintenance safer and reliable, when used in military construction (e.g., greenhouses, farms), also can play the advantages of the intelligent time measurement and adjustment, compared to people more accurately and quickly. In addition, this design also has civilian value. When applied to a family, which can realize the intelligent connection and control of household, provide users with more convenient and comfortable home life.

\section{References}

[1] Mcclain J T, Wimpey B J, Barnhard D H, et al. Distributed robotic target acquisition using Bluetooth communication[C]// Southeast Regional Conference. 2004:291-296.

[2] $\mathrm{Hu} \mathrm{J}, \mathrm{Fu} \mathrm{W}$, Gao S, et al. Implementation of STC15F2K60S2 MCU and CAN bus interface[J]. Microcomputer \& Its Applications, 2014.

[3] Ren Y Y, Niu X. Design of Portable Low Frequency Signal Generator Based on STC15F2K60S2[J]. Journal of Jiyuan Vocational \& Technical College, 2013.

[4] Saptadi A H. Perbandingan Akurasi Pengukuran Suhu dan Kelembaban Antara Sensor DHT11 dan DHT22 Studi Komparatif pada Platform ATMEL AVR dan Arduino[J]. Jurnal Infotel, 2015.

[5] Wang Z H, Bai C Z. Design and Implementation of Multi-point Temperature and Humidity Alarm Systemin Laboratory Based on DHT11[J]. Shanxi Electronic Technology, 2011.

[6] Ni L M, Liu Y, Lau Y C, et al. LANDMARC: indoor location sensing using active RFID[J]. Wireless Networks, 2004, 10(6):701-710. 